\title{
Characteristics Analysis of Short Flux-path 4/3 SRM
}

\author{
Jin-Woo Ahn*
}

\begin{abstract}
This paper presents a design and characteristics analysis of novel 2-phase 4/3 switched reluctance motor (SRM) with short flux path for an air-blower application. The desired air-blower is unidirectional application, and requires a wide positive torque region without torque dead-zone. In order to get a wide positive torque region without torque deadzone during phase commutation, asymmetric inductance characteristic with non-uniform air-gap is considered. The proposed motor could be started at any rotor position with high efficiency drive. The proposed 2-phase 4/3 SRM is verified by finite element method analysis.
\end{abstract}

Keywords: Switched reluctance motor, Short magnetic flux-path, Two-phase, 4/3 pole SRM

\section{Introduction}

Recently, high speed drives are much interested in the industrial applications to reduce the system size and to increase the drive efficiency. Especially, blowers, compressors, pump and spindle drives are suitable for the high speed motor drives, and the demand for the high speed motor system is much increased according to the industrial market. Some types of electric motor such as DC, permanent magnet and reluctance motor have been researched.

SRM has simple structure and inherent mechanical strength without rotor winding and permanent magnet. These mechanical structures are suitable for harsh environments and high temperature and high speed applications ${ }^{[1-4]}$.

This paper presents of a 2-phase 4/3 SRM for an airblower. In this application, the impeller rotates only one direction, and it requires a wide positive torque without torque dead-zone.

The proposed 4/3 SRM has self-starting ability without dead-zone. The 4/3 SRM is employed 4 stator poles and 3 rotor poles. In order to get a wide positive torque region without torque dead-zone during phase commutation, asymmetric inductance characteristic with non-uniform airgap is considered.

The proposed motor is to employ a short flux path. The advantages of short flux path are increasing the efficiency and torque production while decreasing the core loses.

In the paper, a novel $4 / 3 \mathrm{SRM}$ is proposed and the

\footnotetext{
* Dept. of Electrical Engineering, Kyungsung University, Korea. (jwahn@ks.ac.kr)

Received 01 February 2013 ; Accepted 15 February 2013
}

characteristics are analyzed to optimize the magnetic circuit of the motor.

\section{Design of 2-Phase 4/3 SRM}

The design and the basic principles of the novel 2phase 4/3 SRM with short magnetic flux is to be considered. And the FEA analyses of the proposed motor are presented. In order to show the advantages of the proposed motor, a comparison of characteristics of the $4 / 2$ and $4 / 3 \mathrm{SRM}$ is shown. Table I shows the specifications of the proposed 4/3 SRM.

Table 1. Specification of the Proposed 4/3 SRM

\begin{tabular}{|c|c|}
\hline Parameters & Value \\
\hline Output power & $210[\mathrm{~W}]$ \\
\hline Rated torque & $0.2[\mathrm{Nm}]$ \\
\hline Rated speed & $10,000[\mathrm{rpm}]$ \\
\hline Number of stator poles & 4 \\
\hline Number of rotor poles & 3 \\
\hline Bore diameter & $32[\mathrm{~mm}]$ \\
\hline Outer diameter & $90[\mathrm{~mm}]$ \\
\hline Stack length & $33[\mathrm{~mm}]$ \\
\hline Number of turns per phase & $120[\mathrm{turns}]$ \\
\hline Resistance of phase winding & $0.4[\mathrm{Ohm}]$ \\
\hline Unaligned inductance & $2[\mathrm{mH}]$ \\
\hline
\end{tabular}

\subsection{Basic principles of 4/3 SRM}

As known, high electrical frequency causes the core losses in the electric machine. In order to reduce the electrical frequency, reduction of the number of rotor poles is adopted. In this research, the rotor structure must be ensured that the motor can produce continuous 
torque with short magnetic flux and the electrical frequency as low as possible. Considering these two requirements, a rotor structure with three rotor poles was selected.

Fig. 1 shows the 2-phase 4/3 SRM structure. The proposed motor has 4 stator poles and 3 rotor poles. The stator core is separated into two c-cores with two poles each, and there are no steel lamination between these ccores. The stator core is fixed by a plate. Each phase consists of windings on two poles of the c-core, and these windings are connected in serial. With this structure, the magnetic flux flows through the stator core are to be shortest.

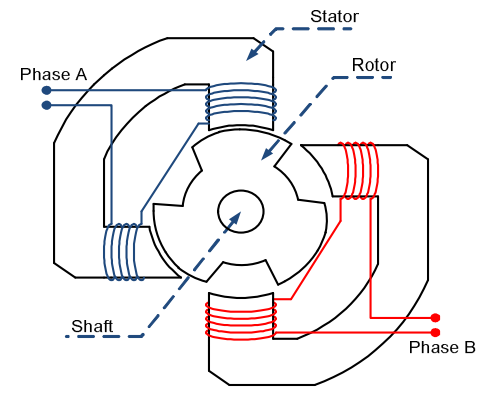

Fig. 1. 2-phase 4/3 SRM structure

The stator poles of the 4/3 SRM are placed at an angle of $120^{\circ}$ that is equal to the rotor pole pitch. And two c-cores are shifted with rotation of $180^{\circ}$.

Electrical frequency of the 4/3 SRM is six times higher than rotation frequency. So, dwell angle is kept of $60^{\circ}$ to produce the continuous torque. In order to get a high torque density, ideal stator pole arc is equal to dwell angle. But there are some manufacturing difficulties, so the stator pole arc was designed with $50^{\circ}$.

The disadvantage of the one-phase and 2-phase motor is self-starting ability, and the 4/3 SRM is not an exception. To overcome this problem, a wider rotor pole arc with nonuniform shape is used. This type of rotor shape is suitable for the applications which require only one rotational direction as air-blowers in this research. The proposed rotor pole arc is $70^{\circ}$. The non-uniform shape is applied for the first $25^{\circ}$ of the rotor poles as shown in Fig. 2.

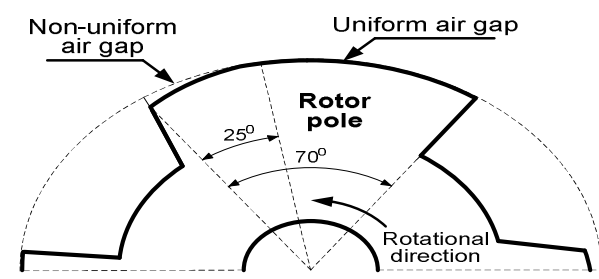

Fig. 2. Rotor shape
The air-gap is determined to develop a flat torque and the rotor shape is stepper type. With geometric structure, the inductance in the motor depends on the air-gap length and overlap area between rotor and stator poles. When the rotor and stator pole are at first overlap region, increasing overlap area results in increasing inductance. Otherwise, when the rotor and stator poles are at full aligned position, the overlap area is constant, reducing of average air-gap between rotor and stator pole results in increasing inductance. So, the motor can produce positive torque during 700 of rotor poles.

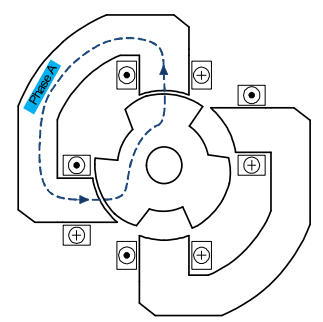

(a)

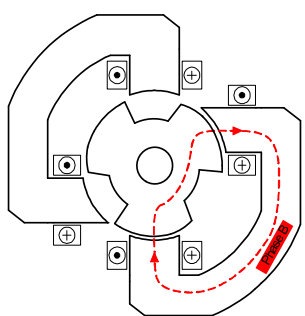

(b)
Fig. 3. Magnetic flux path (a) Phase A; (b) Phase B

Fig. 3 shows the magnetic flux path in the proposed motor at the aligned position. Because of separating of the c-cores, the magnetic flux flows only through the excited poles, and it can't flow through the other poles.

To keep the rotor poles not touching into the stator poles, the stator core is fixed by an aluminum plate. The plate has no influences on flux distribution of the proposed motor.

Fig. 4 shows the full assembly of the proposed 4/3 SRM.

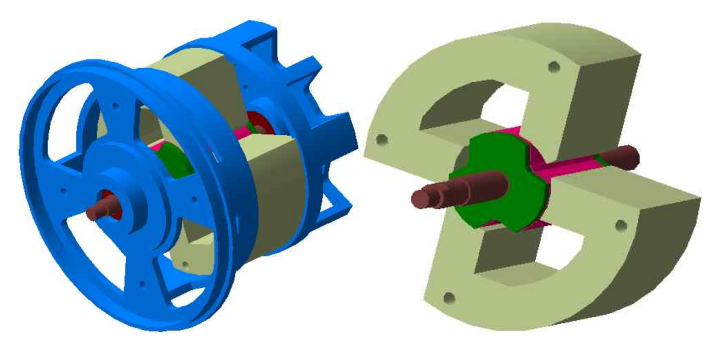

Fig. 4. Full assembly of the 4/3 SRM

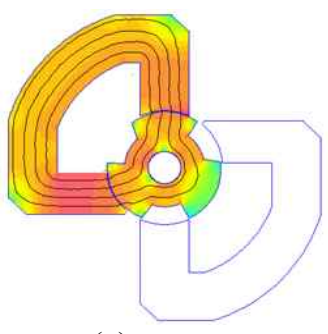

(a)

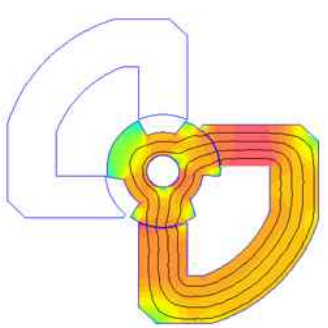

(b)
Fig.. 5. Flux distribution (a) Phase A; (b) Phase B 


\subsection{FEA Analyses}

Fig. 5 shows the flux distribution of the proposed motor. In this paper, the characteristic of the proposed motor is analyzed by using a 2D FEA analysis. By using the FEA software, the characteristic of the flux distribution that influences the performance and characteristic of the motor is analyzed. As shown in Fig. 5, the magnetic flux only flows through the respective excited c-core. Actually, small amount of flux flows through the other, but it is not appreciable.

Torque is one of the important performance parameters of the motor. The higher torque density that means the motor size and the cost can be reduced. Compared with the other motor which have a uniform rotor shape, the proposed motor has high average torque.

Torque characteristics depend on the relationship between flux linkages and rotor position as a function of phase current. Torque varies with rotor position and phase current. The torque production can be estimated by (1).

$$
T_{e}=\frac{1}{2} i^{2} \frac{d L(\theta, i)}{d \theta}
$$

where $T_{e}$ is the electromagnetic torque, $i$ is the excited current, and $L(\theta, i)$ is the inductance dependent on the rotor position and phase current.

By using the 2D FEA, the inductance and torque characteristics are analyzed. Fig. 6 shows the torque profile of the proposed motor. As shown, the overlap torque is about 100. This overlap torque ensures that the motor can be operated without the torque dead-zone and has selfstarting ability.

Fig. 6 shows the inductance profile of the proposed motor. As the stator placement and rotor shape, the inductance profile has curved and symmetric shape.

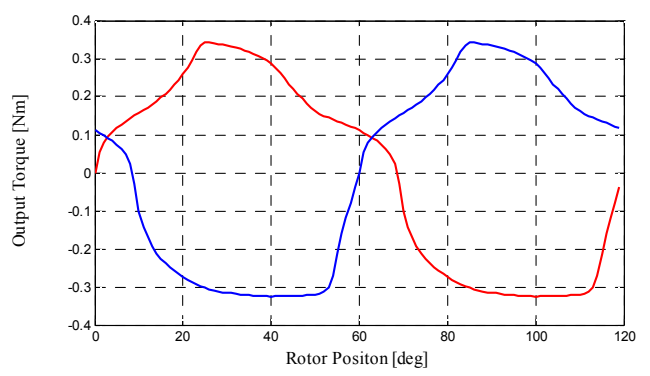

Fig. 6. Torque profile

Fig. 7 shows the torque comparison of the $4 / 2$ SRM and the proposed 4/3 SRM. Both of $4 / 2$ and $4 / 3$ SRM use the same dimension and input parameters. In this analysis, the 4/2 SRM has a stepper rotor shape [8]. In order to compare their torque, the torque profile of $4 / 2$ and $4 / 3 \mathrm{SRM}$ is analyzed in the same electrical position.

As shown in Fig. 8, the average torque of $4 / 3 \mathrm{SRM}$ is $0.21[\mathrm{Nm}]$ while average torque of $4 / 2 \mathrm{SRM}$ is $0.16[\mathrm{Nm}]$. The average torque of the $4 / 3 \mathrm{SRM}$ is $31 \%$ higher than the 4/2 SRM.

\section{Simulation of proposed motor}

Fig. 9 shows the simulation diagram of $4 / 3$ SRM. The simulation diagram consists of the 4/3 SRM model, inverter part, speed and current control part.

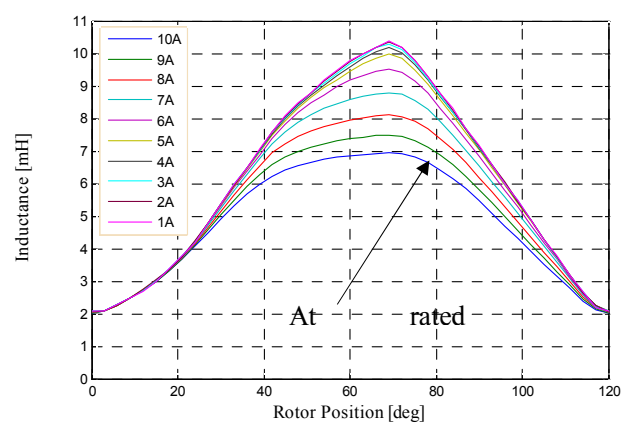

Fig. 7. Inductance profile

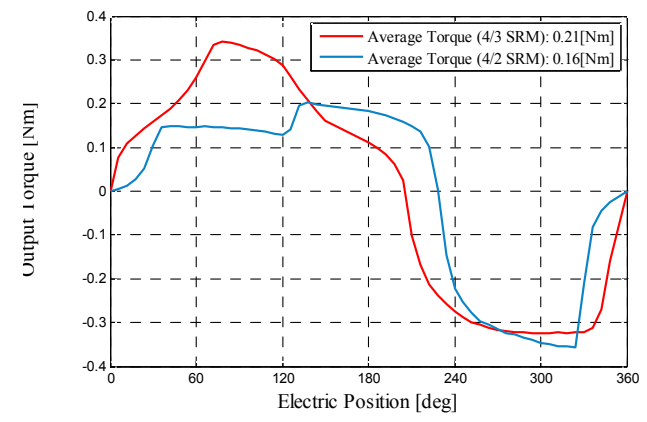

Fig. 8. Torque of the $4 / 2$ and the proposed $4 / 3$ SRM

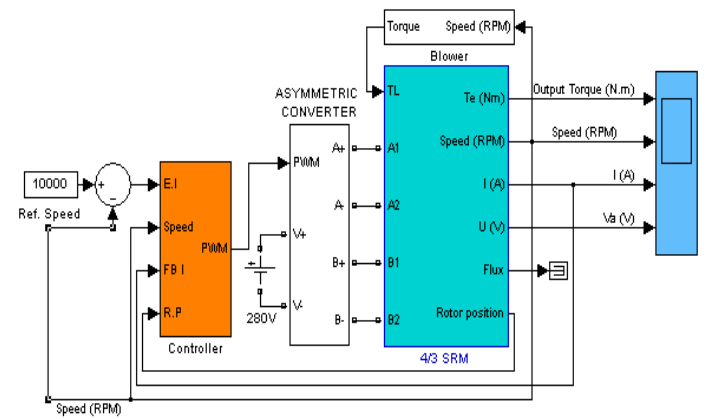

Fig. 9. Simulation diagram of the proposed motor 
After the proposed motor was analyzed by the FEA software, the full torque and inductance were analyzed. The 2D look-up tables of the current verse rotor position and flux linkage and of the torque verse rotor position and current were used for simulation.

The relationship between voltage, resistance and inductance of SRM is given by (2).

$$
v=R_{s} i+\frac{d \lambda(\theta, i)}{d t}
$$

where, $R_{s}$ is the phase resistance, $v$ is the voltage applied across the phase winding, and $\lambda(\theta, i)$ is the phase flux linkages depending on rotor position and excited current.

The voltage drop of the SRM can be determined by using the phase flux linkage data and phase resistance. The speed of the SRM can be determined by using the load equation. The load for simulation consists of inertia, friction and load torque. The load equation is given by (3).

$$
J \frac{d \omega_{m}}{d t}+B \omega_{m}=T_{e}-T_{l}
$$

where $J$ is the combined moment of inertia of the motor and load, $B$ is the combined friction coefficient of the motor and load, $T_{l}$ is load torque.

From (3) the speed of SRM is determined by (4).

$$
\omega_{m}=\int \frac{T_{e}-T_{l}-B \omega_{m}}{J} d t
$$

The number of turns per stator poles is only one in the FEA analysis. After that, the number of turns is determined by observing tail of current when it is turned off. High turn number results in low current/torque ratio and long tail of current and vice versa. Low current/torque ratio is good index for performance whereas long tail of current will produce negative torque. Balancing these two things, 50 turns/pole was selected.

As shown in Fig. 10 (a), the speed rising time is about $0.6 \mathrm{~s}$, it is fast enough for system response. The speed rising time depends on the unaligned inductance, current slope, and the system inertia moment. The lower unaligned inductance results in the lower speed rising time while rated current and voltage are kept constant. In order to reduce the unaligned inductance, the turn number of the windings can be reduced while increasing the excited current, and keeping the mmf is constant.
Fig. 10 (b) shows the simulation results of 4/3 SRM after adjusting the zoom. In this simulation, the operational parameters are the same with real system. The proposed motor is operated at $10,000 \mathrm{rpm}$ with excited current is $8 \mathrm{~A}$ and the supplied dc voltage is $280 \mathrm{~V}$.

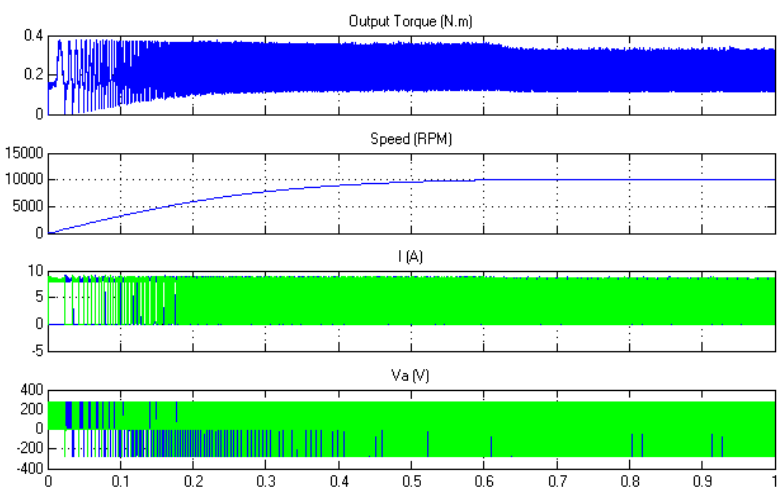

(a)

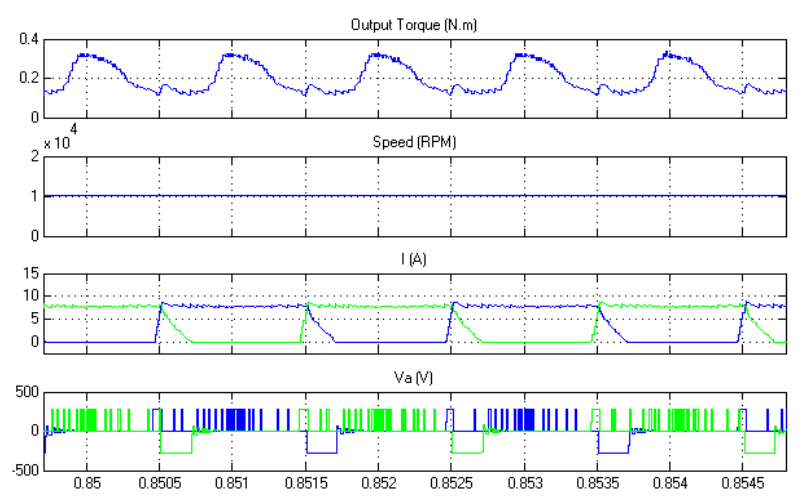

(b)

Fig. 10. Simulation results of the proposed motor

\section{Conclusion}

This paper presents a novel 2-phase 4/3 SRM which has short flux path to reduce core losses and increase torque production. In order to produce a continuous output torque, the positive torque region is extended with asymmetric inductance characteristic. The air-gap is also determined to reduce torque ripple. The proposed motor is suitable for applications that require unidirectional rotation and is easy to manufacturing because of simple stator shape.

\section{Acknowledgment}

This work was supported by Kyungsung University Research Grant in 2013. 


\section{References}

[1] R. Krishnan, "Switched Reluctance Motor: Modeling, Simulation, Analysis, Design, and Applications", CRC Press, 2001.

[2] T.J.E Miller, "Switched Reluctance Motor and Their Control", Magna Physics Publishing, Aug 1, 1993.

[3] T. Genda, H. Dohmeki, "Characteristics of $4 / 2$ Switched Reluctance Motor for a high speed drive by the excitation angle", International Conference on Electrical Machines and Systems, ICEMS 15-18, pp.1-6, Nov 2009.

[4] D. H. Lee, H. K. M. Khoi, J. W. Ahn, "Design and Analysis of High Speed 4/2 SRMs for an air-blower", IEEE International Symposium Industrial Electronics, pp.1242-1246, July 2010.

[5] Tanujaya, M, D. H. Lee, J. W. Ahn, "Design and Analysis of a Novel 4/5 Two-Phase Switched Reluctance Motor", IEEE International Conference of Electrical Machines and System, pp. 1-6, Aug 2011.

[6] Tanujaya, M, D. H. Lee, J. W. Ahn, "Characteristic analysis of a Novel 6/5 c-core type three-phase Switched Reluctance Motor", IEEE International Conference of Electrical Machines and System, pp. 1-6, Aug 2011.

[7] Cheewoo Lee, Krishnan, R, "New Designs of a TwoPhase E-Core Machine by Optimizing of Magnetic Structure for a Specific Application: Concept, Design, and Analysis", IEEE Industry Applications Society, Vol. 45, No. 5, pp. 1804-18014, Sept 2009.

[8] Dong-Hee Lee, Jin-Woo Ahn, "Performance of High Speed 4/2 Switched Reluctance Motor," Journal of Electrical Engineering \& Technology, vol. 6, no. 5, pp.640 646, 2011.

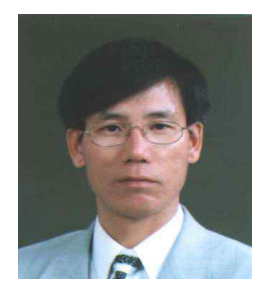

Jin-Woo Ahn received his B.S., M.S., and Ph.D. degrees in Electrical Engineering from Pusan National University, Pusan, Korea, in 1984, 1986, and 1992, respectively.

He has been with Kyungsung University, Busan, Korea, as a professor in the Department of Mechatronics Engineering since 1992. He was a visiting researcher in the Speed Lab at Glasgow University, U.K., a visiting professor in the Dept. of ECE and WEMPEC at the University of Wisconsin-Madison, USA, and a visiting professor in the Dept. of ECE at Virginia Tech from July 2006-June 2007. He was the director of the Advance Electric Machinery and Power Electronics Center and the President of Korea Regional Innovation System Association. He also has been the director of the Smart Mechatronics Advanced Research and Training Center from Aug. 2008 to July 2011 and the Senior Easy Life Regional Innovation System since July 2008, which are authorized by the Ministry of Knowledge Economy, Korea. He is the author of five books including SRM, the author of more than 150 papers and has more than 20 patents. His current research interests are advanced motor drive systems and electric vehicle drives. He has been the Editor-in-Chief of JICEMS, Chairman of ICEMS2013 and IEEE ICIT2014.

Dr. Ahn received several awards including the Best Paper Award from the Korean Institute of Electrical Engineers in 2002 and 2011, The Korean Federation of Science and Technology Society in 2003, Korean Institute of Power Electronics in 2007, Park Min-Ho Prize in 2009, Busan Science \& Technology Prize in 2011 and Ministerial Citation of Ministry of Knowledge Economy in 2011, respectively. He is a Fellow of the Korean Institute of Electrical Engineers, a member of the Korean Institute of Power Electronics and a senior member of the IEEE. 